single cells, not merely populations of cells." This requires further development of the technology. For CGAP, having the funds and the ability to bring in a wider community of technology developers to work on these problems should expedite this process.

Brendan Horton is on the staff of Nature, based in

Washington.e-mail:b.horton@naturedc.com

\section{Visionaries seek UK national strategy}

\section{Alison Mitchell}

The Imperial Cancer Research Fund (ICRF) has a vision. By 2020, it believes that considerable advances will have been made in the prevention, early detection and treatment of cancer. But it cannot achieve these goals alone and, last month, it supported calls for a national strategy on cancer research - a collaboration between the UK government, the research charities and industry.

At present, charity research institutions cannot freely apply to government research agencies for support. Paul Nurse, directorgeneral of the ICRF, says: "Government funding needs to be more flexible, to ensure that resources are spent where they can be best used." The stumbling block seems to be that government grant-awarding bodies allocate only a small percentage of their budgets to cancer research precisely because the

\begin{tabular}{l}
\hline Table 3 Sites of Internet interest for UK cancer research \\
\hline Site name \\
General \\
\hline ICRF
\end{tabular}

cancer charities exist. But links are being forged between the charities and industry, and such schemes may provide opportunities for students, postdocs and group leaders.

The ICRF is the largest UK cancer charity, spending $\mathfrak{E} 54$ million (US\$89 million) a year on research, closely followed by the Cancer Research Campaign (CRC) which spends

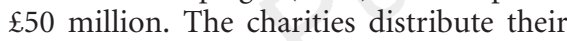
funds very differently. The ICRF runs its own laboratories, while the CRC mainly supports research within universities and medical schools (see box below). How much room do these strategies leave for collaboration?

Plenty, it seems. Because of the way it distributes its funds, the CRC is particularly open to joint research ventures. As well as funding large groups within centres such as

\title{
Research opportunities at the cancer charities
}

Research at the ICRF is broken down into basic science, clinical studies and translational research - the middle ground between the two. Most of the basic science is carried out at the laboratories in central London and at Clare Hall in

Hertfordshire.

These sites employ around 80 graduate students and 180 postdocs. Students usually receive funding for four years from the charity, and must register externally for $\mathrm{aPhD}$.

About 30 postdoctoral fellowships are available each year in basic research. For those bringing their own grants, the ICRF offers access to support services (including cell and media production, oligonucleotides, instruments and antibodies). Recruitment of group leaders has slowed after big campaigns in the last two years, but positions are available.

Clinical research units are based in hospitals and medical schools around the United Kingdom, covering areas ranging from immunotherapy to breast oncology. A total of 33 graduate students is funded by the ICRF, plus 44 clinical fellows and 49 postdocs.

The CRC divides its research between basic and clinical studies in a roughly 45:55 split. It awards grants to groups at the Paterson Institute in Manchester, the Beatson Institute in Glasgow, the Institute for Cancer Research and associated CRC centres, the Gray Laboratory Cancer Research Trust in London, and groups at universities such as Birmingham and Dundee.

Seventy-six students are funded by the CRC. Training follows guidelines set by the host institutions.

The CRC runs three fellowship schemes Research Fellowships for Clinicians, Senior Clinical Fellowships and Senior Cancer Research Fellowships. Between them, these support 597 postdoctoral fellows. Scientists can also apply for project grants, which are reviewed by the grants committee four times a year. The Wellcome/CRC Institute operates junior and senior research groups, with the junior groups staying for a maximum of ten years.

Both charities are developing 'translational research', to bridge the gap between basic and clinical research. The ICRF has a training scheme in this area, which is now in its third year, with ten clinical or postdoctoral fellows. the Institute of Cancer Research in London, in 1989 the CRC was one of the partners that set up the Wellcome/CRC Institute in Cambridge. The Wellcome Trust does not fund cancer research, but the scientific focus at this institute is on basic research into the cellular processes that are relevant to development. The ICRF's clinical directorate also has strong research partnerships with the National Health Service trusts. Mike Probert, the charity's assistant director of research, clinical division, says most of the clinical studies "would not be possible without the flow of patients through NHS hospitals".

The charities are also increasingly looking for ways to develop their discoveries commercially. The ICRF's technology transfer arm, Imperial Cancer Research Technology (ICRT), bridges the gap between basic research and product development. Last October, in a joint venture with the pharmaceutical company Antisoma, ICRT announced a phase III clinical trial of Theragyn. This drug is based on a murine monoclonal antibody developed by ICRF scientist Joyce Taylor-Papadimitriou and colleagues. The antibody is chemically linked to a radioactive isotope, yttrium-90, which is targeted to cancer cells. Phase I/II trials showed it to be particularly effective in patients with ovarian cancer.

\section{Marketing teamwork}

The CRC's technology transfer company is Cancer Research Campaign Technology (CRCT) which announced last month that it was teaming up with the UK company Serotec to market immunological reagents developed by the CRC. Another venture is Oncotech, which aims to develop the commercial potential of cancer research. This is a consortium of CRCT, the Leukaemia Research Fund, the law firm Cameron McKenna and the patent firm Mewburn Ellis.

So, in many ways, a national strategy for cancer research may not be very far away. Indeed, the ICRF and CRC already belong to a joint venture with the Medical Research Council and the Department of Health. But cancer research encompasses many areas of 
basic science - from cell growth and death to DNA repair and replication - as well as clinical trials, epidemiology and medical statistics. The ICRF and CRC cover all these areas, but there are also many smaller, more specialist charities. These many institutions have one common aim, however - to turn scientific advances into effective ways to prevent or treat cancer.

Alison Mitchell is on the staff of Nature, based in

London.e-mail:a.mitchell@nature.com

\section{Therapeutics: a glimpse of the future}

\section{Owen Goldring}

Cancer therapeutics has, until recently, been out of fashion - being seen as an applied rather than an academic discipline. But that is changing, thanks to advances in the understanding of the molecular nature of cancers, coupled with the hot debate centred around telomerase and the initial unravelling of the mechanisms of angiogenesis. A radical new generation of anti-cancer drugs should ensue.

The focus is on discovering small-molecule mechanism-based inhibitors that selectively target or starve cancers. There is a belief that there will be a first generation of ras farnesylation inhibitors, receptor tyrosine kinase inhibitors, and possibly p53 drugs, in the next five years ( rasfarnesylation and EGF receptor tyrosine kinase inhibitors are already in phase I clinical trials). Almost up with those will be the first angiogenesis inhibitors. The predictions are that cell-cycle inhibitors will be the next, with apoptosis after that. Telomerase inhibitors may come within five or ten years.

But, given that cancer is basically a disease of an unstable genome, some scientists believe that cancer will develop resistance to any drug thrown at it. They feel that more research is needed now to look at resistance mechanisms.

\section{Search for selective drugs}

There will be no sudden development of a 'pan-cancer' drug — the opposite will probably be true, with selective molecular drugs of high therapeutic index being used against specific solid tumours. Neither will conventional anti-cancer drugs, such as methotrexate and cyclophosphamide, be replaced overnight. But every researcher would like to offer a treatment that is more selective and considerably less toxic.

Drug discovery programmes are using techniques such as random screening for finding chemical leads, or structural rational design, where structural biologists use NMR and crystallography to figure out the molecular interaction between a target and an inhibitor (see Anti-Cancer Drug Design 12, 525-531; 1997). These methods require a new breed of cancer scientist.

Paul Workman, the new director of the CRC Centre for Cancer Therapeutics, at the Institute of Cancer Research's labs in Surrey in the United Kingdom, says that, before the advent of molecular oncology, there was a cultural, ideological and skill-capability misfit between cancer biologists and those making derivatives of methotrexate. These two schools did not speak the same language. Now, they are beginning to, he says.

So, what skills do you need if you want to get into cancer therapeutics today? Workman says the type of scientist that biotech companies and even some major pharmaceutical companies lack is what he calls the "cancer pharmacologist". "These are not 'general' pharmacologists who are brought into a cancer project, as occurs in some companies. They are people who live and breathe cancer pharmacology, understand cancer pathways and can apply their pharmacological skills to these new molecular opportunities. They will not necessarily have cloned genes, but I do expect them to be able to do PCR, western blots, northerns, Southerns and so on." They need to understand the language of molecular biology, and traditionally trained pharmacologists tend not to have these skills, Workman says.

Two types of people are needed: those who have done biological science degrees, molecular biology, biochemistry, and then learn pharmacology; and those trained as traditional pharmacologists, because they bring a familiarity with concepts such as dose-response relationships and kinetics, says Workman. "Most molecular biologists have no training in these critical aspects of pharmacology. You need both. And you also need people trained in pharmacokinetics."

\section{Angiogenesis link to mutation?}

Each tumour needs to develop its own blood supply, says Roy Bicknell, head of the Imperial Cancer Research Fund's angiogenesis lab in the Institute of Molecular Medicine at John Radcliffe Hospital, Oxford. "Small tumours remain dormant until they undergo an angiogenic switch, when they start making angiogenic factors, and then recruit in new blood vessels. The tumour then grows rapidly. There is evidence to suggest the angiogenic switch is linked to a mutation in p53," says Bicknell.

Bicknell apparently does not mind which areas of science his $\mathrm{PhD}$ students come from: "They must be bright and inquisitive but, as long as they are practically adept, we reckon we can fill in any gaps in theoretical knowledge." One of his students has a first-class degree from Cambridge in zoology, another is a veterinarian from Germany, and a third has a medical degree from Oxford University.

Another, somewhat controversial, area of cancer therapeutics is telomerase inhibition. About $85 \%$ of tumour cells produce telomerase, which keeps telomeres stable, and the assumption has been that cancer cells need telomerase to maintain continuous cell division.

Geron, a company based in Menlo Park, California, is studying the genetic clock of cell ageing, telomeres and telomerase. Geron has a substantial programme of research on telomerase inhibition (see Table 1).

\section{Bioengineering angle}

One possible route for future work on telomerase and angiogenesis is offered by Martin Braddock, leader of the endothelial gene expression group in the vascular diseases unit at Glaxo Wellcome's Stevenage labs in southern England: "When we expose endothelial cells in culture to fluid shear stress (for example, mimicking what happens with arterial blood flow), we have evidence that functional telomerase activity is downregulated." He encourages people to think about coupling telomerase activity with biomechanical forces, and relating that to cellular processes in angiogenesis.

"I think that's the way angiogenesis is going to go in the future," he says, adding that it will be understood almost from a bioengineering angle rather than from a purely cell or molecular biological angle. "We're almost at the start of the era of tissue engineering."

Cancer therapeutics is an area where the opportunity for doing exciting and groundbreaking research is very much on the up. But one problem that researchers will face at least in Europe - is the dearth of second postdoc positions to enable them to get greater experience and a step further up the ladder to landing that elusive permanent academic job.

Many high-flyers gravitate from Europe to the United States after a first postdoc, do more academic research and then sometimes spend time in the biotech industry before returning to Europe. They are often welcomed back with open arms, being seen as having demonstrated an entrepreneurial outlook as well as having enhanced their academic experience.

Few people automatically turn to industry straight after a $\mathrm{PhD}$ or postdoc, perceiving that in industry people have to change subjects often. But this may be a misconception. Glaxo's Braddock says: "In our own particular area of research at the moment we are productive, and I hope that will remain so. If we hadn't been productive over the last few years we wouldn't work on it. But if we were in a university lab and non-productive, and had no grants to write after three years, the same situation would apply."

Owen Goldring is a UK-based freelance science/medical writer and industrial consultant. e-mail:ogoldring@edit1.demon.co.uk. 